\title{
NUMERICAL SIMULATION OF THE STRESS-STRAIN STATE OF COMPLEX-REINFORCED ELEMENTS
}

\author{
Olena KRANTOVSKA, Mykola PETROV, Liubov KSONSHKEVYCH, Matija OREŠKOVIĆ, Sergii SYNII, Nelli ISMAILOVA
}

\begin{abstract}
The article describes a developed technique of a numerical simulation of the stress-strain state of complex-reinforced elements, which allows you to create models of double-span continuous. The performed experimental and theoretical studies allowed us to carry out the testing of the developed design model and to justify the reliability of the proposed numerical simulation methodology. The results of the experimental studies were compared with those of the theoretical studies. The theoretical calculus algorithm was developed by using the finite element method. Theoretical calculations were performed by using the mathematical-graphical environment software system LIRA-SOFT and the mathematical and computer program MATLAB. On the basis of the experimental research, the iso-fields of displacements and stresses in the materials of an eccentrically compressed beam with a small bend of the slab were constructed, which collapse behind the inclined narrow strip of concrete and displacements and stresses in the materials of the eccentrically stretched beam, which is destroyed due to the yield of the upper mounting armature.
\end{abstract}

Keywords: deformation model; finite element modelling; LIRA-SOFT; stress-strain state

\section{INTRODUCTIONS}

One of the prominent factors in the design of beamreinforced concrete elements in a complex stressed state is the resistance of the supporting areas, which remains unexplained due to the static uncertainty of this task.

The execution of experimental and theoretical studies with the aim of improving the existing and developing new, modern calculation models of complex stressed supporting areas of reinforced concrete elements is an important and actual task. At the same time, the application of the theory of plasticity, methods of the mechanics of deformation and destruction of the solid allow us to describe in detail the process of the deformation of experimental reinforced concrete elements.

The purpose of the study-development, based on the experimental and theoretical research, was numerical modelling of the stress-strain state (SSS) of complicated elements using numerical nonlinear finite-element calculations.

Today, the finite element method (FEM) [1] has taken the top position because of its obvious advantages, the possibility of bringing the problem to a system of linear or nonlinear algebraic equations.

The theory of M. I. Karpenko [2-4] showed high efficiency in the programmable modeling of the work of reinforced concrete with cracks based on the FEM, which were generalized by the V. I. Murashov theory for the case of a complex stressed state [5]. Works by L. Prandtl [6], E. Ries [7], A. A. Ilyushin and many others were devoted to the studies of the plasticity of materials with their complex SSS.

The theory of the plasticity of concrete and reinforced concrete proposed in [1] was substantially developed by A. I. Kozachevsky [8], V. M. Kruglov [9], S. F. Klovanich [10], V. I. Korsun [11] and others. Hereafter, M. I. Karpenko and his students developed the theory of small elastic-plastic deformations, in which concrete before and after the appearance of cracks is considered to be anisotropic.

\section{RESEARCH METHODOLOGY \\ 2.1 General Guidelines}

In accordance with the developed methodology of experimental research, the items listed below have been tested:

- $\quad$ single-span beams of a rectangular cross-section with the dimensions of $100 \times 200 \mathrm{~mm}$ with $h_{0}=175 \mathrm{~mm}$, span $l$ $=9 \cdot h_{0}=575 \mathrm{~mm}$ and shear span $a / h_{0}=1,2,3$, made of the heavy concrete of classes C12/15, C20/25, C30/35, reinforced by two plain frames with the lower rebar $2 \varnothing 12,14,16$ A500S, with the top rebar $2 \varnothing 8,10,12$ A500S and with the transverse rebar $2 \varnothing 3,4,5 \mathrm{~V}_{\mathrm{r}}-\mathrm{I}$;

- two-span continuous beams, respectively, $b \times h=100 \times$ $180 \mathrm{~mm}$ with $h_{0}=155 \mathrm{~mm}, l=8 \cdot h_{0}, a / h_{0}=1,2,3$, $\mathrm{C} 12 / 15, \mathrm{C} 20 / 25, \mathrm{C} 30 / 35,2 \varnothing 10,12,14500 \mathrm{~S}, 2 \varnothing 10,12$, 14 A500S, 2ø3, 4, 5 V $\mathrm{r}-\mathrm{I}$.

Single-span beams were tested for eccentrically stretching at $N_{\mathrm{st}} /\left(f_{\mathrm{ck}} \cdot b \cdot h_{0}\right)=0.05 ; 0.2 ; 0.35$, eccentric compression $N_{\mathrm{st}} /\left(f_{\mathrm{ck}} \cdot b \cdot h_{0}\right)=0.2 ; 0.4 ; 0.6$, in a numerical experiment, modelled using a deformation method based on the results of previous field experiments, on eccentric compression with $N_{\mathrm{st}} /\left(f_{\mathrm{ck}} \cdot b \cdot h_{0}\right)=0.05 ; 0.2 ; 0.35$. The eccentricity of the application of the longitudinal force was changed: $e / h_{0}=-0.25 ; 0 ;+0.25$. The continuous same beams were tested on the bending by two concentrated forces.

For the experiment, power plants were manufactured according to the schemes [12].

Experiments were performed on five-factor, close to the properties of the D-optimal plan of type N5, which provides the same accuracy of the prediction of the experimental value in the area described by a radius equal to 1 relative to the central "0" point (Experiment No. 27), as well for two-factor, 
three-level, close to the properties of the D-optimal, plan B2 type for the study of eccentrically stretched beams [13].

\subsection{Modelling of the Stress-Strain State of Reinforced Concrete Beams}

Concrete and reinforcement in the experimental samples work together, but have different physical and mechanical properties characterized by the non-linear dependence between the stresses and strains. In this regard, for the implementation of finite-element models of experimental reinforced concrete elements in the LIRA-SOFT PC, additionally, the tested complete concrete deformation diagrams were introduced according to the data of the NIISK (A. M. Bambura) and two-line diagrams of the reinforcement $[14,15]$.

Calculations were made by only one-half of the beam due to the symmetry of the experimental elements and loads. The beams were conditionally broken into spatial octogenic iso parametric finite elements No. 236 with the dimensions of $1 \times 1 \times 1 \mathrm{~cm}$ for the convenience of the modelling of the reinforcement, and considering that in real beams, a large aggregate was crumbled by granite gravel fraction $5-10 \mathrm{~mm}$. The number of elements in the model of the acontinuous beam were - 19600, nodes - 22725; the conventional and eccentrically compressed beam, respectively, 15800 and 18320.

The calculations used a stepwise and stepwise-iterative method using the piecewise linear dependence of the library number 14 according to the corresponding algorithm: if the value of the generalized deformation goes beyond the given fractured line, then the exclusion of the material $\left(E_{i}=1\right)$ of the elementary plane from the cross section is modeled. In the performance of real and numerical experiments, at first stages, eccentrically compressed, stretched load was applied, and then - transverse load in the form of two symmetrically located concentrated forces.

The adapted layered model of the deformation of the experimental elements and the execution of numerical experiments is realized in the MATLAB PC environment. According to [16], we adopt a deformation model of the beam in a discrete form: divide it into i-sections and j-layers up the height of a cross-section. Let us assume that the formed sites have the same SSS. The reinforcement of the stretched and compressed zones is tied to the cross-sectional faces at the level of the centres of gravity. The basis of the SSS simulation of the experimental beams is the traditional position for the deformation model.

The modelling of the SSS of the experimental beams is performed by the following algorithm:

1) Initial information is entered.

2) The geometric characteristics of the beam's crosssections are determined by taking into account the real modulus of the deformation of cross sections at this stage:

$$
(E A)_{r e d, i}=\sum_{j=1}^{j \max }\left(E_{c i j} A_{c i j}+E_{s i} A_{s i}+E_{s i}^{\prime} A_{s i}^{\prime}\right)
$$

$$
\begin{aligned}
& (E S)_{r e d, i}=\sum_{j=1}^{j \max }\left(E_{c i j} A_{c i j} y_{i j}+E_{s i} A_{s i} y_{s i}+E_{s i}^{\prime} A_{s i}^{\prime} y_{s i}^{\prime}\right) \\
& y_{0 i}=\frac{(E S)_{r e d, i}}{(E S)_{r e d, i}} \\
& (E I)_{r e d, i}=\sum_{j=1}^{j \max }\left[E_{c i j} A_{c i j}\left(y_{0 i}-y_{i j}\right)^{2}+\right. \\
& \left.+E_{s i} A_{s i}\left(h_{0}-y_{0 i}\right)^{2}+E_{s i}^{\prime} A_{s i}^{\prime}\left(y_{0 i}-a^{\prime}\right)^{2}\right]
\end{aligned}
$$

where $E_{c i j}(t), E_{s i}, \quad E_{s i}^{\prime}-$ cross-sectional deformation modules, respectively concrete, stretched and compressed reinforcement, which before the load are accepted as equal to the initial deformation modules; $A_{c i j}$ - area of the $j^{\text {th }}$ layer of the $i^{\text {th }}$ section of the beam; $y_{i j}-$ distance from the compressed side of the beam to the centre of the gravity of the $j^{\text {th }}$ layer of the $i^{\text {th }}$ section of the beam.

3) The initial coordinate of the pole of the longitudinal force $N$ is determined.

$$
y_{P_{0}}=\left[\frac{h}{2}-y_{0 i} \pm\left(e+e_{0 a c .}\right)\right]
$$

where $e$ - eccentricity of applying a longitudinal stretching or compression force; $e_{0 a c}$. - accidental eccentricity,

$e_{0 a c .}=\frac{h}{30}$

4) The stage of the load is given with the subsequent increase in the given magnitude of the longitudinal compressive (stretching) force $\Delta N=0.1\left[N_{a d m}\right]$ to a given or admissible value. For a continuous element, we define the transverse force with its subsequent increase on $\Delta V$, and determine the corresponding spans and support moments and the transverse forces on the medium support. Before the appearance of a plastic hinge on the medium support, these moments are counted on a nondeformed scheme, according to the methods of construction mechanics.

The support and span moments are accepted:

$$
\begin{aligned}
& M_{s u p}=-0,236 \mathrm{~V} ; M_{s u p}=-0,205 \mathrm{~V} ; M_{s u p}=-0,130 \mathrm{~V} \\
& M_{s p}=0.143 \mathrm{~V} ; M_{s p}=0.079 \mathrm{~V} ; M_{s p}=0.022 \mathrm{~V} .
\end{aligned}
$$

At this stage of the work of a continuous beam, the value of the transverse force on the medium support to the right and to the left of it is determined, respectively:

$$
V_{\text {sup }, \text { right }}=\frac{M_{s p}-M_{\text {sup }}}{a}
$$


$V_{\text {sup,left }}=\frac{M_{\text {sup }}-M_{s p}}{a}$

With the appearance of a plastic hinge over the middle support, the support moment reaches the maximum possible value of $M_{\text {sup }}=\left[M_{\text {sup }}\right]$, which can be sustained by the concrete cross-section over the support. Furthermore, there is a redistribution of internal forces with increasing span moments, which are:

$M_{s p}=\frac{V \cdot d \cdot a-M_{s u p} \cdot d}{L} \leq\left[M_{s p}\right]$

where $\left[M_{s p}\right]$ - the maximum admissible value of the span moment; $d$ - the distance from the first support to the line of action of the concentrated force; $a$ - is the distance from the line of action of the concentrated force to the medium support.

The transverse force on the support is determined after the appearance of a plastic hinge:

$V_{u l t}=\frac{\left[M_{s p}\right]-\left[M_{\text {sup }}\right]}{a}$

5) The deformations of concrete and reinforcement in the sections of the beam are determined:

$$
\begin{aligned}
& \varepsilon_{c i j}=\frac{N}{(E A)_{\text {redi }}} \cdot\left[1+\frac{\left.y_{P}\left(y_{i j}-y_{0 i}\right)(E A)_{\text {redi }}\right]+\frac{M_{i}\left(y_{i j}-y_{0 i}\right)}{(E I)_{\text {redi }}}(13)}{(E I)_{\text {redi }}}\right] \cdot\left[1+\frac{y_{P}\left(h-a_{i}-y_{0 i}\right)(E A)_{\text {redi }}}{(E I)_{\text {redi }}}\right]+\frac{M_{i}\left(h-a_{i}-y_{0 i}\right)}{(E I)_{\text {redi }}}(14) \\
& \left.\varepsilon_{s i}=\frac{N}{(E A)_{\text {redi }}}\right] \\
& \varepsilon_{s i}^{\prime}=\frac{N}{(E A)_{\text {redi }}} \cdot\left[1+\frac{y_{P}\left(a_{i}^{\prime}-y_{0 i}\right)(E A)_{\text {redi }}}{(E I)_{\text {redi }}}\right]+\frac{M_{i}\left(a_{i}^{\prime}-y_{0 i}\right)}{(E I)_{\text {redi }}}(15) \\
& y_{P i}=y_{P_{0}}+y_{N(x)},
\end{aligned}
$$

where $y_{N}(x)$ - additional deflection or bend of the $i^{\text {th }}$ section, due to the longitudinal force $N$, which is determined by the equation:

$$
y_{N(x)}=\left[\frac{\left(\varepsilon_{s i}-\varepsilon_{b 1}^{\prime}\right)}{h_{0}}\right] \cdot \rho_{m N i} \cdot l^{2}
$$

where $\rho_{m N i}-$ a coefficient that takes into account the effect of the moment on the longitudinal force $N$ (or the effort of the previous tension) on the magnitude of its bend or buckling. It is determined by the length of the beam $(0 \leq x \leq 1)$ by the equation $\rho_{m N i}=\frac{l \cdot x-x^{2}}{2 l^{2}}$

6) If deformations in concrete and reinforcement do not exceed the maximum admissible values, then we remember them. Through this value, we determine the stresses according to the diagrams of the $\sigma-\varepsilon$ concrete and reinforcement. Then we perform the correction of the cross-section modules of concrete deformation and reinforcement.

$$
\begin{aligned}
& E_{c i j}=\frac{\sigma_{c i j}}{\varepsilon_{c i j}} \\
& E_{s_{i, l o w}}=\frac{\sigma_{s i, l o w}}{\varepsilon_{s i, l o w}} \\
& E_{s_{i, u p}}=\frac{\sigma_{s i, u p}}{\varepsilon_{s i, u p}}
\end{aligned}
$$

Next, we determine the geometric characteristics of the beams' cross-sections for the specified values of the modules of the deformation of the cross-sections $(E A)_{\text {red }, i},(E I)_{\text {red, }, \text {. }}$

7) Through the corrected geometric characteristics, specify the values of the deformations of concrete and reinforcement and compare them with the previous values. If their values are close (no more than $1 \%$ inaccuracy), then at this stage, the SSS of the beams is revealed and you can go to the definition of deflections and other parameters of the workability of the experimental beams.

8) According to the author's improved technique [14], we determine the size of the shear bearing capacity of the inclined cross-sections $\left[V_{u l t}\right]$ of the experimental reinforced concrete beams.

9) After reaching the given or admissible value of the longitudinal stretch force $\left[N_{a d m}\right]$, we turn to the consideration of the change of the SSS in the process of the proportional loading of a single-span beam by symmetrically disposed transverse forces $F$ to the destruction of the element per a normal or inclined crack.

10) Determine the values of the bending moment and transverse force along the length of the beam.

11) Through the Eqs. (13)-(16), we determine the deformation of concrete and reinforcement, and also specify the coordinate of the pole of the force $\left[N_{a d m}\right]$, taking into account an additional bending due to the action of transverse and longitudinal forces,

$$
y_{P i}=y_{P_{0}}+y_{t o t, i}
$$

where $y_{\text {tot }, i}-$ a complete deflection, which is defined as the algebraic sum of deflections due to bending deformations from the transverse and longitudinal loading, as well as the shear deformations: 


$$
y_{t o t, i}=\left(\frac{\varepsilon_{s i}-\varepsilon_{c 1}}{h_{0}}\right) \cdot\left(\rho_{m N i}+\rho_{m M i}\right) l^{2} \cdot \rho_{q i}
$$

where $\rho_{m M i}$ - a coefficient that takes into account the influence of the bending moment on the deflection of the beam in this cross section. It is counted within the shear span.

12) At each stage, the values of the increasing transverse force $V$ near the medium support for the two-span beam and the destructive force $V_{u l t}$ are compared. If $V \geq V_{u l t}$, then this means that the external load has reached a value at which the beam can be destroyed on the medium support section along the inclined cross-section.

\section{RESULTS}

The results of the numerical implementation of the experiments of an ordinary one-span (test task), eccentrically stretched and compressed, as well as the continuous reinforced concrete beams (five-factor experiments) are presented in Tab. 1.

Table 1 Destructive loading of experimental elements

\begin{tabular}{|c|c|c|c|c|c|c|c|c|c|}
\hline \multirow[b]{2}{*}{ №№ } & \multicolumn{3}{|c|}{ Eccentrically-compressed beams } & \multicolumn{3}{|c|}{ Eccentrically stretched beams } & \multicolumn{3}{|c|}{ Continuous double-span beams } \\
\hline & $V_{l}^{\text {exp. }}$ & LIRA-SOFT & MATLAB & $V_{l}^{\exp .}$ & LIRA-SOFT & MATLAB & $V_{l}^{\exp }$ & LIRA-SOFT & MATLAB \\
\hline 1 & 77 & 82 & 77 & 19,5 & 22 & 21 & 118 & 130 & 112 \\
\hline 2 & 117 & 123 & 120 & 43,1 & 53 & 45 & 140 & 140 & 121 \\
\hline 3 & 124 & 127 & 123 & 136,7 & 140 & 135 & 103 & 115 & 95 \\
\hline 4 & 57 & 57 & 54 & 48,8 & 51 & 49 & 60 & 70 & 50 \\
\hline 5 & 135 & 142 & 137 & 32,0 & 48 & 34 & 193 & 205 & 165 \\
\hline 6 & 69 & 70 & 69 & 13,8 & 23 & 16 & 91 & 100 & 61 \\
\hline 7 & 65 & 70 & 62 & 54,4 & 56 & 54 & 64 & 80 & 60 \\
\hline 8 & 106 & 114 & 106 & 155,3 & 102 & 146 & 82 & 90 & 85 \\
\hline 9 & 140 & 145 & 140 & 65,2 & 79 & 78 & 139 & 150 & 171 \\
\hline 10 & 50 & 58 & 49 & 13,5 & 22 & 9 & 83 & 90 & 88 \\
\hline 11 & 63 & 71 & 62 & 42,8 & 52 & 43 & 88 & 95 & 62 \\
\hline 12 & 108 & 112 & 110 & 122,6 & 128 & 121 & 118 & 130 & 80 \\
\hline 13 & 144 & 150 & 146 & 143,3 & 145 & 131 & 165 & 170 & 169 \\
\hline 14 & 54 & 58 & 54 & 48,5 & 52 & 49 & 68 & 85 & 62 \\
\hline 15 & 59 & 62 & 57 & 9,8 & 10 & 3 & 93 & 105 & 90,5 \\
\hline 16 & 104 & 111 & 105 & 54,0 & 70 & 67 & 99 & 115 & 92 \\
\hline 17 & 50 & 66 & 60 & 38,3 & 44 & 37 & 83 & 95 & 85 \\
\hline 18 & 133 & 137 & 123 & 101,6 & 102 & 102 & 130 & 145 & 145 \\
\hline 19 & 95 & 101 & 97 & 55,7 & 63 & 55 & 100 & 110 & 115 \\
\hline 20 & 81 & 88 & 79 & 55,6 & 61 & 55 & 80 & 80 & 76 \\
\hline 21 & 93 & 97 & 91 & 59,5 & 62 & 59 & 91 & 105 & 101 \\
\hline 22 & 84 & 89 & 86 & 51,3 & 57 & 51 & 89 & 100 & 88 \\
\hline 23 & 96 & 102 & 93 & 18,0 & 27 & 20 & 102 & 105 & 112 \\
\hline 24 & 85 & 92 & 88 & 76,9 & 70 & 77 & 78 & 85 & 86 \\
\hline 25 & 102 & 105 & 93 & 50,1 & 53 & 50 & 104 & 105 & 109 \\
\hline 26 & 74 & 87 & 83 & 60,0 & 66 & 61 & 76 & 85 & 97 \\
\hline 27 & 89 & 92 & 89 & 55,4 & 62 & 55 & 90 & 100 & 99 \\
\hline \multicolumn{2}{|c|}{$v=\frac{\sigma}{b_{0}} \cdot 100 \%$} & 12 & 5.7 & & 18 & 6.4 & & 11.8 & 6.6 \\
\hline
\end{tabular}

The use of this finite element calculation allows you to simulate the SSS of test beams at all stages of operation, up to destruction.

A sequential analysis of the iso-fields of the displacements and stresses (Figs. 1,2) in the materials of real construction allows us to estimate the influence of the investigated factors and the factors of external influence on their bearing capacity, to predict the character of further deformations and destruction.

It was found that the stresses in transverse reinforcement reach, on average, $58 \%$ of the yield strength. Due to the twoaxial compression of the concrete in the supporting area, strengthening is observed (in relation to the prism), on average, it is $22 \%$, and its bearing capacity, respectively, is
$43 \%$. After the formation of the so-called "plastic hinge" over the medium support of the continuous beam with an increasing load, there is a known redistribution of moments (within the limits of $20 \ldots 30 \%$ ) with the growth of the latter in the spans and the destruction of their supporting areas is completed by the achievement of all transverse reinforcement of the yield limit. The destruction, practically, of all continuous double-span beams occurred due to the punching shear over the middle support with the achievement of compressive stresses in concrete under the concentrated forces and over the support, respectively, $\sigma_{c x} \cong 0.72 f_{c k}$ and $1.03 f_{c k}, \sigma_{c z} \cong 0.96 f_{c k}$ and $0.66 f_{c k}, \tau_{c x z} \cong 0.47 f_{c k}$ with $x=l-$ a...l. 

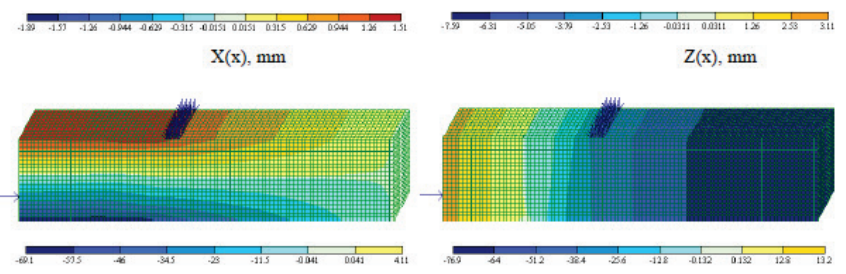

$\sigma_{\mathrm{cs}, \mathrm{MPa}}$
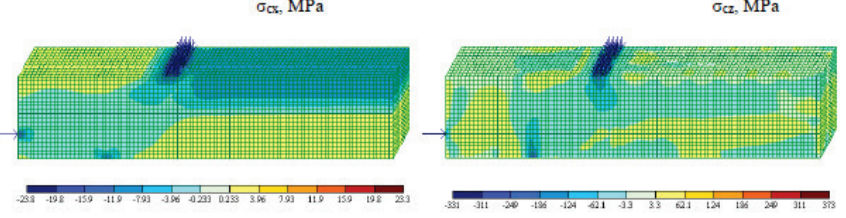

$\tau_{\mathrm{zz}}, \mathrm{MPa}$
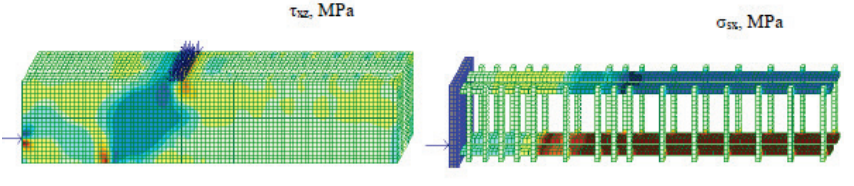

$\sigma_{\mathrm{sz}}, \mathrm{MPa}$

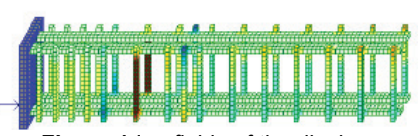

Figure 1 Iso-fields of the displacement and stresses in the materials of an eccentrically compressed beam with a small shear span which collapses per inclined compressed concrete strip (Experiment 8).
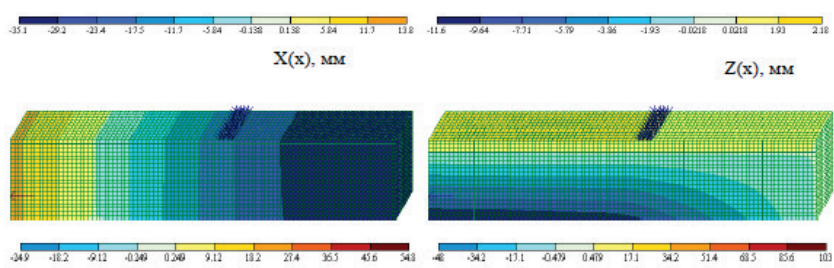

$\mathrm{Z}(\mathrm{x}), \mathrm{MM}$

$\sigma_{\mathrm{cx}}, \mathrm{MPa}$
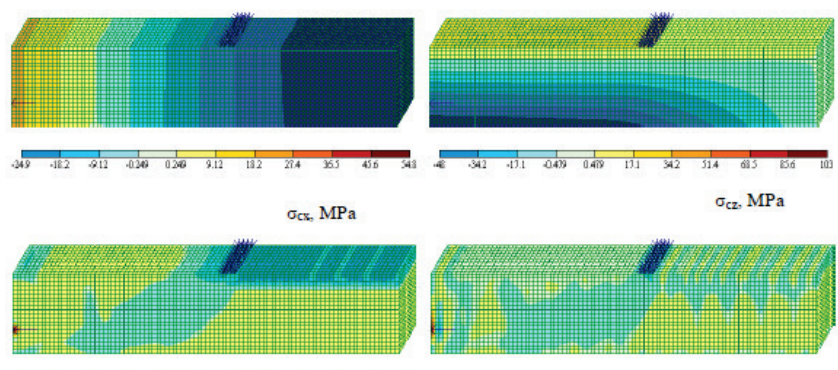

$\sigma_{\mathrm{cz}}, \mathrm{MPa}$

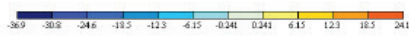

$\tau_{\mathrm{xz}}, \mathrm{MPa}$

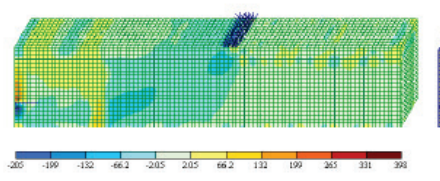

$\sigma_{\mathrm{sz}}, \mathrm{MPa}$

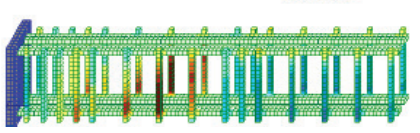

Figure 2 Iso-fields of the displacement and stresses in the materials of an eccentrically stretched beam which is destructed by the yielding flow of the upper reinforcement (Experiment 2).

\section{CONCLUSIONS}

The numerical modelling methodology of the SSS of complex-reinforced concrete elements with the help of a numerical nonlinear finite-element calculation based on the general mechanics of reinforced concrete is developed. The finite-element calculation was used to model the stressstrain state of the experimental beams at all stages of operation, up to destruction.

The adequacy of this method is confirmed by its comparison with the results of the conducted field experiments to determine the bearing capacity of an ordinary single-span, eccentrically stretched and compressed beam, as well as continuous reinforced concrete beams. Research have found that the stresses in armatures reach $58 \%$ of the limit of release. In the support area, the reinforcement averaged $22 \%$ and its bearing capacity was $43 \%$.

The destruction of all continuous double-span beams occurred due to the punching shear over the middle support with the achievement of compressive stresses in the concrete. Theoretical calculations by using the PC LIRASOFT and MATLAB showed convergence with the experimental data in the range of the coefficient of variation of $6 \ldots 11.8 \%$.

\section{REFERENCES}

[1] Klovanich S. F. \& Mironenko I. N. (2007). Method of Finite Elements in the Mechanics of Reinforced Concrete, Izd-o ONMU, Odessa.

[2] Gvozdev, A. A. \& Karpenko, N. I. (1965). The work of reinforced concrete with cracks under a plane stressed state. Structural Mechanics and Analysis of Constructions, 2, 20-23.

[3] Karpenko, N. I. (1996). General models of mechanics of reinforced concrete. Stroyizdat, Moscow.

[4] Karpenko, N. I. \& Klovanich, S. F. (1983). Determining relations for reinforced concrete with cracks in thermosensitive effects. Structural Mechanics and Analysis of Constructions, 2, 6-11.

[5] Gorodetsky, A. S., Shmukler, V. S., \& Bondarev, A. V. (2003). Information technologies for calculating and designing building constructions. NTU "KhPI", Kharkiv.

[6] Prandtl, L. (1924). Spannungsverteilung in plastischen Körpern. Proc. of $1^{\text {st }}$ Int. Congr. of Appl. Mech., 43-54.

[7] Reiss, E. (1948). Accounting for elastic deformation in the theory of plasticity. Theory of plasticity, 206-222.

[8] Kozachevsky, A. I. (1983). Modification of the deformation theory of plasticity of concrete and the plane stress state of reinforced concrete with cracks. Structural Mechanics and Analysis of Constructions, 4, 12-16.

[9] Kruglov, V. M. (1983). Nonlinear relations and criteria for the strength of concrete in a triaxial stress state. Structural Mechanics and Analysis of Constructions, 4, 12-16.

[10] Klovanich, S. F. (2004). The finite element method in nonlinear mechanic soil and concrete. Building construction, 61(1), 103-108.

[11] Korsun, V. I. (2005). Design constructions for temperature and force effects taking into account the inhomogeneity of the properties of materials, dis. Dr. tech. Sciences, DonNACEA, Makeevka.

[12] Krantovska, O., Petrov, M., Ksonshkevych, L., Synii, S., \& Sunak, P. (2018). Improved engineering method for calculating the strength of the supporting areas of reinforced concrete elements. MATEC Web of Conferences, Kharkiv. https://doi.org/10.1051/matecconf/201823002014

[13] Voznesensky, V. A. (1981). Static methods of experiment planning in technical and economic research. Finance and Statistics, Moscow. 
[14] Dorofeev, V. S., Karpiuk, V. M., \& Krantovska, E. N. (2010). Strength, crack resistance and deformability of continuous reinforced concrete beams (monograph). Even, Odesa.

[15] Dorofeev, V. S., Karpiuk, V. M., \& Petrov, N. N. (2011). Strength, deformability and crack resistance of the support of inclined sections of eccentrically stretched, compressed of reinforced concrete beams (monograph). Even, Odesa.

[16] Blikharskyy, Z. Y. (2005). Onset-deformation of the millconcrete constructions in the aggressive medium during the day, dis. Dr. tech. Sciences, LPNU, Lviv.

\section{Authors' contacts:}

\section{Olena KRANTOVSKA}

Odessa State Academy of Civil Engineering and Architecture,

Ditrikhson st. 4, 65029 Odesa, Ukraine

Tel: +380660474510

\section{Mykola PETROV}

Odessa National Academy of Telecommunications O. S. Popov, Kovalska st. 1, 65029 Odesa, Ukraine

Tel: +380677630679

\section{Liubov KSONSHKEVYCH}

Odessa State Academy of Civil Engineering and Architecture,

Ditrikhson st. 4, 65029 Odesa, Ukraine

Tel: +380669170688

\section{Matija OREŠKOVIĆ}

University North, Department of Civil Engineering,

48000 Koprivnica, Croatia

E-mail: moreskovic@unin.hr

\section{Sergii SYNII}

Lutsk National Technical University, Lvivska st. 75, 43018 Lutsk, Ukraine

E-mail: sergii.synii@gmail.com

\section{Nelli ISMAILOVA}

Odessa National Academy of Telecommunications O. S. Popov, Kovalska st. 1, 65029 Odesa, Ukraine

Tel: +385508545585 